\title{
No gender-related bias in COPD diagnosis and treatment in Sweden: a randomised, controlled, case-based trial
}

\author{
Hamid Akbarshahi (1) ${ }^{1}$, Zainab Ahmadi ${ }^{1}$, David C. Currow ${ }^{2}$, Jacob Sandberg (1) ${ }^{1}$, \\ Zac Vandersman ${ }^{2}$, Aaron Shanon-Honson ${ }^{2}$ and Magnus Ekström (1) ${ }^{1,2}$
}

Affiliations: ${ }^{1}$ Dept of Clinical Sciences, Division of Respiratory Medicine and Allergology, Lund University, Lund, Sweden. ${ }^{2}$ IMPACCT, Faculty of Health, University of Technology Sydney, Ultimo, Australia.

Correspondence: Hamid Akbarshahi, Dept of Clinical Sciences, Division of Respiratory Medicine and Allergology, Lund University, SE-221 00 Lund, Sweden. E-mail: hamid.akbarshahi@med.lu.SE

\section{ABSTRACT}

Introduction: COPD is a major cause of morbidity and mortality. The prevalence, morbidity and mortality of COPD among females have increased. Previous studies indicate a possible gender bias in the diagnosis and management of COPD. The present study aims to determine if there is gender bias in the management of COPD in Sweden.

Methods: This was a double-blind, randomised (1:1), controlled, parallel-group, web-based trial using the hypothetical case scenario of a former smoker (40 pack-years and quit smoking 3 years ago) who was male or female. The participants were blind to the randomisation and the purpose of the trial. The case progressively revealed more information with associated questions on how the physician would manage the patient. Study participants chose from a list of tests and treatments at each step of the case scenario.

Results: In total, 134 physicians were randomised to a male $(n=62)$ or a female $(n=72)$ case. There was no difference in initial diagnosis (61 (98\%) male cases and 70 (97\%) female cases diagnosed with COPD) and planned diagnostic procedures between the male and female cases. Spirometry was chosen by all the physicians as one of the requested diagnostic tests. The management of the hypothetical COPD case did not differ by sex of the responding physician.

Conclusion: In Sweden, diagnosis and management of a hypothetical patient with COPD did not differ by the gender of the patient or physician.

@ERSpublications

No gender-related bias in COPD management https://bit.ly/3gTsWoG

Cite this article as: Akbarshahi $\mathrm{H}$, Ahmadi Z, Currow DC, et al. No gender-related bias in COPD diagnosis and treatment in Sweden: a randomised, controlled, case-based trial. ERJ Open Res 2020; 6: 00342-2020 [https://doi.org/10.1183/23120541.00342-2020].

This article has supplementary material available from openres.ersjournals.com.

The study was prospectively registered at www.ClinicalTrials.gov with identifier number NCT02728674. The deidentified database can be made available upon reasonable request by contacting the corresponding author.

Received: 2 June 2020 | Accepted after revision: 18 Aug 2020

Copyright $\odot$ ERS 2020. This article is open access and distributed under the terms of the Creative Commons Attribution Non-Commercial Licence 4.0. 


\section{Introduction}

COPD is a devastating and severe disease that is more frequent among males than among females. In a population-based study including subjects aged $\geqslant 40$ years in the Netherlands, the incidence of COPD was higher in men (3.54 cases per 1000 person-years) than in women (2.34 cases per 1000 person years) [1-3]. However, the prevalence, morbidity and mortality of COPD among females have increased [4], mainly due to changes in occupational exposure and smoking habits in the population [5-9]. Mortality rates among male COPD patients have declined, while the hospitalisation and death rates among female patients in the USA have not changed significantly [10]. Likewise, an analysis of the Spanish National Hospital Database of patients admitted with acute exacerbations of COPD as their primary diagnosis showed a significant reduction in mortality of male patients with COPD from 2006 to 2014, but not among females [11].

There are gender differences among patients with COPD regarding impact on function and morbidity. Pulmonary function impairment is greater in female smokers compared to male smokers for the same level of tobacco exposure, generating a greater susceptibility to development of COPD [12]. Women experience worse quality of life and more severe breathlessness for the same level of objective pulmonary compromise compared to male smokers [2]. Furthermore, $80 \%$ of people with COPD who have no history of smoking are female [8]. Moreover, women may suffer from a different phenotype of COPD by being more susceptible to developing chronic bronchitis and having more breathlessness and anxiety [13]. These clinical differences between men and women further emphasise the importance of prompt recognition of possible cases, suitable diagnostic procedures, accurate assessment of the severity of the disease and appropriate treatment [8]. There is also a difference between men and women with COPD regarding the functional status, where men had better scores than women regarding total score, domestic and leisure domains of the London Chest Activity of Daily Living [14].

A survey of primary-care physicians showed that a diagnosis of COPD was less likely to be considered in women than in men even with identical symptoms and physical examination [5]. Gender-related bias in the diagnosis of COPD may compromise the proper diagnosis and treatment of the disease in females $[5,15,16]$.

Knowledge of the role of gender inequalities in healthcare is lacking. Gender biases can lead to systematic underdiagnosis and undertreatment of both female and male patients, depending on the condition [17, 18].

The primary aim of this study was to test the hypothesis that the clinical decision-making process of the physicians for the proposed diagnosis differs between a male and female hypothetical patient with identical COPD symptoms using a random case survey. Secondary aims were to explore whether the clinical decision-making process differed by gender of the responding physician.

\section{Methods}

\section{Study design}

This was a double-blind, randomised (1:1), controlled, parallel-group, web-based trial using hypothetical case scenarios. The study was approved by the Lund University Research Ethics Committee (Dnr: 2015/596). All participants gave their informed consent. The study was prospectively registered with ClinicalTrials.gov (Nr: NCT02728674) and is reported in accordance with the Consolidated Standards of Reporting Trials (CONSORT) guidelines [19]. Study procedures have been described elsewhere [20]. The study design included two different randomisations for the symptom and gender of the patient: chronic breathlessness versus chronic pain; and male versus female patient. Participants were not aware of the randomisation or the purpose of the trial.

\section{Procedures}

Physicians were recruited through the Swedish Respiratory Society, the Swedish Society of Internal Medicine, the Swedish Society of Primary Care, the Swedish Society of Palliative Medicine and in the authors' departments (internal medicine, pulmonary medicine and primary care) using an e-mail with general information about the study (an anonymous survey on the management of a patient with respiratory problems in clinical practice). Participants answered the eligibility criteria and were asked to consent to participate.

Inclusion criteria were: licensed physician; treating patients with respiratory problems in clinical practice; able to read and understand a case description in Swedish; not on the research team and not aware of the study's design or content; and no previous participation in the study. The participants logged in using their Swedish identification number to avoid entry more than once.

Data were collected on the participant's age, gender, professional seniority, present location of practice, specialty license(s), current specialty area(s) of practice and number of years working as a physician. All the questions and case scenarios are available in the supplementary material (appendix S1). 
Randomisation and case scenario

Participating physicians were randomised to a case scenario of a former smoker (40 pack-years and quitted smoking 3 years ago) who was male or female. The participants were blind to the randomisation or the purpose of the trial. The clarity, consistency, face validity and clinical plausibility of the case scenarios and study questions were piloted by a sample of physicians $(n=10)$ who did not subsequently participate in the main survey and were refined based on this testing. The technical function of the web-based questionnaire was also optimised.

The case progressively revealed more information with associated questions on how the physician would diagnose and treat the patient (supplementary appendix S1). Study participants chose from a list of tests and treatments at each step of the case scenario. The participant had to answer each question in order to advance to the next page and each page was separately saved before proceeding. The participant could not return to or change earlier responses. They were requested to answer the questions in accordance with how they would manage the patient in their clinical practice.

\section{End-points}

The study end-points were assessed in three stages: 1) the most probable diagnosis (asthma, cancer, heart failure, COPD, chronic pulmonary emboli or other; primary end-point); 2) diagnostic procedures required to make the diagnosis (spirometry, blood test, electrocardiogram, exercise testing, echocardiography, $\mathrm{O}_{2}$ saturation levels or sputum culture; secondary end-point); and 3) treatment offered (short-acting bronchodilator, long-acting anticholinergic inhaler, long-acting $\beta_{2}$-agonist inhaler, both long-acting anticholinergic and $\beta_{2}$-agonist inhalers, inhaled corticosteroids, triple therapy, oral corticosteroids, respiratory rehabilitation treatment, consultation with a dietitian or oxygen therapy; secondary end-point).

\section{Sample size}

The original protocol was designed to address the need of symptomatic treatment of chronic breathlessness and chronic pain in a hypothetical COPD case [20]. In the absence of previous evidence, we assumed that the same differences would exist regarding COPD diagnosis between male and female patients in the case.

\section{Statistical analyses}

Categorical data were tabulated using frequencies and percentages. The categorical end-points were analysed between groups using Chi-squared tests and logistic regression. The influence of the gender of the responding physician on the end-points was evaluated by introducing an interaction term between the gender of the physician and the patient in the logistic models. Estimates were reported as odds ratios with $95 \%$ confidence intervals. The agreement between male and female physicians was analysed using $\kappa$ coefficient.

Statistical significance was defined as $\mathrm{p}<0.05$. Analyses were conducted with Stata version 14.2 (StataCorp LP, College Station, TX, USA).

\section{Results}

From September 2016 to May 2017, 134 physicians were randomised to a male $(n=62)$ or a female $(n=72)$ case. Of these physicians, $53 \%$ were male, $51 \%$ worked in a hospital setting and $46 \%$ worked in primary care. Characteristics of the physicians were well balanced between the groups (table 1).

The initial diagnosis and planned diagnostic procedures were similar between the male and female cases (table 2). Spirometry was chosen by all the physicians as one of the requested diagnostic tests. Echocardiography was chosen as one of the diagnostic procedures in female patients nearly twice as often as in male patients (11 versus 5$)$ although this did not reach statistical significance $(p=0.20)$.

In the next stage, physicians were asked to propose a management plan. All the participants suggested a short-acting $\beta_{2}$-agonist and a long-acting anticholinergic inhaler as the recommended initial treatment, while oxygen therapy was not chosen by any of the physicians (table 3). There was no difference between male and female patients when it came to the proposed treatment plan for COPD. In the next step, participants were asked to manage the patient following the given scenario: the patient comes for a new appointment after 3 months with essentially unchanged symptoms; the patient is treated with the triple treatment and has undergone 8 weeks of respiratory rehabilitation. Our data showed no gender difference regarding the proposed follow-up intervention (changing the inhaler, intensifying respiratory rehabilitation treatment, benzodiazepines, opioids, oral steroids, Roflumilast or oxygen therapy) by the physicians (table 3 ).

The diagnosis and diagnostic management did not differ by sex of either the case or the responding physician. All the physicians in both respective groups suggested spirometry as a diagnostic approach 


\begin{tabular}{|c|c|c|c|}
\hline Characteristic & All & Male case & Female case \\
\hline Physicians n & 134 & 62 & 72 \\
\hline Female & $63(47)$ & $24(38)$ & $39(62)$ \\
\hline \multicolumn{4}{|l|}{ Professional seniority } \\
\hline Resident & 43 (32) & $20(15)$ & $23(17)$ \\
\hline Specialist & 52 (39) & $24(18)$ & $28(21)$ \\
\hline Senior specialist & $38(28)$ & $18(13)$ & $20(15)$ \\
\hline \multicolumn{4}{|c|}{ Present location of practice } \\
\hline Hospital & $69(51)$ & $33(25)$ & $36(27)$ \\
\hline Primary healthcare & $62(46)$ & $27(20)$ & $35(26)$ \\
\hline Other & $6(4)$ & $3(2)$ & $3(2)$ \\
\hline \multicolumn{4}{|l|}{ Specialty licence(s) ${ }^{\#}$} \\
\hline Internal medicine & 50 (37) & $26(19)$ & $24(18)$ \\
\hline Family medicine & $62(46)$ & $27(20)$ & $35(26)$ \\
\hline Respiratory medicine & $32(24)$ & $13(10)$ & $19(14)$ \\
\hline Other & $31(23)$ & $19(14)$ & $12(9)$ \\
\hline \multicolumn{4}{|c|}{ Current specialty area(s) of practice $\#$} \\
\hline Internal medicine & $36(27)$ & $19(14)$ & $17(13)$ \\
\hline Primary care & $61(46)$ & 26 (19) & $35(26)$ \\
\hline Respiratory medicine & $32(24)$ & $13(10)$ & $19(14)$ \\
\hline Other & $24(18)$ & $14(10)$ & $10(7)$ \\
\hline
\end{tabular}

(table 4). Further analyses did not show any bias for male physicians managing male/female cases. The same applied for female physicians.

We analysed the influence of gender of the responding physician on the end-points by introducing an interaction term between the gender of the physician and the patient in the logistic models. There were too few cases (and no difference) between male and female respondents to check for interaction for the primary end-point (COPD diagnosis). The analyses for the other end-points did not show any significant interaction except for the blood tests ( $\mathrm{p}$-value 0.007).

We investigated the agreement between male and female physicians, which shows $57.46 \%$ agreement (expected 49.78\%) - к: 0.1530 (SE: 0.0856).

\section{Discussion}

In the present study we found no gender-related difference in evaluation and management of a hypothetical patient with COPD. Furthermore, the analysis for responding physician gender did not indicate any significant difference in the process of diagnostic approach between female and male physicians.

Conversely, previous studies of primary care physicians in North America and Spain have shown a gender-biased diagnosis and management of a hypothetical COPD case [5, 15]. Differences in study design

\section{TABLE 2 Diagnosis and evaluation of a male versus female patient with COPD}

\begin{tabular}{lccc} 
& Male patient & Female patient & p-value \\
\hline Cases n & 62 & 72 & \\
COPD diagnosis & $61(98)$ & $70(97)$ & 0.649 \\
Spirometry & $62(100)$ & $72(100)$ & $>0.999$ \\
Blood test & $45(73)$ & $52(72)$ & 0.963 \\
ECG & $42(68)$ & $50(69)$ & 0.832 \\
Exercise testing & $7(11)$ & $8(11)$ & 0.974 \\
Echocardiography & $5(8)$ & $11(15)$ & 0.199 \\
$\mathbf{O}_{2}$ saturation levels & $49(68)$ & 0.813 \\
Sputum culture & $41(66)$ & $2(3)$ & 0.186 \\
\hline Data are presented as $\mathrm{n}(\%)$, unless otherwise stated. & & \\
\hline
\end{tabular}




\section{TABLE 3 Management of a male versus female patient with COPD}

\begin{tabular}{|c|c|c|c|}
\hline & Male patient & Female patient & p-value \\
\hline Cases $\mathbf{n}$ & 62 & 72 & \\
\hline Short-acting bronchodilator & $62(100)$ & $72(100)$ & $>0.999$ \\
\hline Long-acting anti-cholinergic inhaler & $62(100)$ & $72(100)$ & $>0.999$ \\
\hline Long-acting $\boldsymbol{\beta}_{2}$-agonist inhaler & $14(23)$ & $27(38)$ & 0.062 \\
\hline Both long-acting anti-cholinergic and $\boldsymbol{\beta}_{2}$-agonist inhalers & $32(52)$ & $40(56)$ & 0.648 \\
\hline ICS & 14 (23) & $17(24)$ & 0.888 \\
\hline Triple therapy & $19(31)$ & $26(36)$ & 0.504 \\
\hline OCS & $10(16)$ & $9(13)$ & 0.548 \\
\hline Respiratory rehabilitation treatment & $46(74)$ & $54(75)$ & 0.915 \\
\hline Consultation with a dietitian & 45 (73) & $48(67)$ & 0.459 \\
\hline Oxygen therapy & $0(0)$ & $0(0)$ & 1.0 \\
\hline Changing the inhaler ${ }^{\#}$ & $4(6)$ & $5(7)$ & 0.910 \\
\hline Intensifying respiratory rehabilitation treatment ${ }^{\#}$ & $4(6)$ & $12(17)$ & 0.069 \\
\hline Benzodiazepines" & $1(2)$ & $0(0)$ & 0.279 \\
\hline Opioids $\#$ & $6(10)$ & $9(13)$ & 0.605 \\
\hline Oral steroids $\#$ & $5(8)$ & $3(4)$ & 0.342 \\
\hline Roflumilast (Daxas) ${ }^{\#}$ & $4(6)$ & $2(3)$ & 0.305 \\
\hline Oxygen therapy ${ }^{\#}$ & $2(3)$ & $0(0)$ & 0.125 \\
\hline
\end{tabular}

Data are presented as $\mathrm{n}(\%)$, unless otherwise stated. ICS: inhaled corticosteroids; OCS: oral corticosteroids. " : follow-up management.

might explain this discrepancy between the studies. In the present study COPD was diagnosed by more than $97 \%$ of the physicians, but the COPD diagnosis rate was lower in the other two studies $[5,15]$. It is possible that the difficulty level of our hypothetical case was lower than that in the other studies. The study by Miravitlles et al. [15] was designed in such a way that dependent on the diagnostic process, it could give rise to eight different cases. In the study by CHAPMAn et al. [5], the case was presented in six versions differing only in the sex and age of the patient. The difference in the study design can be considered a contributing factor to the lower rate of COPD diagnosis in those studies.

A recent epidemiological study demonstrated that COPD is now equally prevalent among men and women in Sweden [21]. Furthermore, there has been a lot of information and focus on physicians' education regarding COPD in Sweden in recent years, and the effects of it can be observed in the results of our study.

The gender distribution among the responding physicians in our study was almost equal (47\% female), while fewer than $20 \%$ of physicians were female in the previous study by CHAPMAN and colleagues [5]. It is unclear if this difference plays a role in the management bias, although we did not observe any bias relative to the physician sex.

In terms of the number of participating physicians, we included a smaller number (134) compared to the above-mentioned studies (192 and 839) [5, 15].

TABLE 4 Diagnostic management of a patient with COPD by a male versus female physician

\begin{tabular}{lccc} 
& Male physician & Female physician & p-value \\
\hline Cases n & 71 & 63 & \\
COPD diagnosis & $69(97)$ & $62(98)$ & 0.631 \\
Spirometry & $71(100)$ & $63(100)$ & 1.0 \\
Blood test & $47(66)$ & $50(79)$ & 0.089 \\
ECG & $45(63)$ & $47(74)$ & 0.162 \\
Exercise ECG & $6(8)$ & $9(14)$ & 0.285 \\
Echocardiography & $8(11)$ & $8(12)$ & 0.799 \\
$\mathbf{0}_{2}$ saturation levels & $45(63)$ & $45(71)$ & 0.322 \\
Sputum culture & $0(0)$ & $2(3)$ & 0.130 \\
\hline Data are presented as $\mathrm{n}(\%)$, unless otherwise stated. & & \\
\hline
\end{tabular}


In our study, spirometry was ordered as frequently for the female as the male patients by both female and male physicians. This is different from previous reports in other countries where spirometry was less frequently ordered for female patients in comparison to male patients [5, 22-24].

Different aspects of gender differences in COPD patients have been studied more frequently in recent years. Female patients develop COPD at earlier ages with lower tobacco consumption in comparison to males [4], and their quality of life is more impaired [25]. Females have an increased susceptibility to COPD due to sex-related biological factors [26] and may have different responses to medical treatment, follow-ups, smoking cessation interventions and pulmonary rehabilitation programmes [27-32]. Women with COPD exacerbations are younger, smoke more actively and have less comorbidity [33]. Nevertheless, the diagnosis and management guidelines for female patients are the same as those for male patients.

A strength of the present study is that a randomised blinded design was employed where participants were unaware that there was randomisation and of the actual purpose of the study. We also provided the physicians with the diagnostic and management options that are in accordance with the most recent international guidelines (Global Initiative for Chronic Obstructive Lung Disease 2019) [34].

A limitation of this work is the lack of data from real practice. However, well-designed clinical vignette questionnaires are highly useful to determine whether clinical decision-making is influenced by the gender of the medical professional and their patients [35].

In conclusion, our data did not support any gender bias in the management of COPD patients.

Acknowledgements: The authors thank the Swedish Respiratory Society, the Swedish Society of Internal Medicine and the Swedish Society of Primary Care for help with standardising recruitment, and all physicians who participated and made this trial possible.

Support statement: This study was supported by The Swedish Respiratory Society, Svenska Läkaresällskapet, Hjärt-Lungfonden and The Scientific Committee of Blekinge County Council. Funding information for this article has been deposited with the Crossref Funder Registry.

Author contributions: H. Akbarshahi had full access to all the data in the study, and takes full responsibility for the integrity of the data and the accuracy of the data analysis. Conception: M. Ekström; design: A. Shanon-Honson, M. Ekström, Z. Ahmadi, Z. Vandersman; acquisition of data: J. Sandberg, M. Ekström, Z. Ahmadi; analysis of data: M. Ekström, Z. Ahmadi, H. Akbarshahi; interpretation of data: all authors; drafting the first version of article: H. Akbarshahi; revision for important intellectual content and approval of the version to be published: all authors.

Conflict of interest: None declared.

\section{References}

1 Ancochea J, Miravitlles M, Garcia-Rio F, et al. Underdiagnosis of chronic obstructive pulmonary disease in women: quantification of the problem, determinants and proposed actions. Arch Bronconeumol 2013; 49: 223-229.

2 Han MK, Postma D, Mannino DM, et al. Gender and chronic obstructive pulmonary disease: why it matters. Am J Respir Crit Care Med 2007; 176: 1179-1184.

3 Afonso AS, Verhamme KM, Sturkenboom MC, et al. COPD in the general population: prevalence, incidence and survival. Respir Med 2011; 105: 1872-1884.

4 Aryal S, Diaz-Guzman E, Mannino DM. COPD and gender differences: an update. Transl Res 2013; 162: $208-218$. Chapman KR, Tashkin DP, Pye DJ. Gender bias in the diagnosis of COPD. Chest 2001; 119: 1691-1695.

6 Barnes PJ. Sex differences in chronic obstructive pulmonary disease mechanisms. Am J Respir Crit Care Med 2016; 193: 813-814.

7 Camp PG, Goring SM. Gender and the diagnosis, management, and surveillance of chronic obstructive pulmonary disease. Proc Am Thorac Soc 2007; 4: 686-691.

8 Cote CG, Chapman KR. Diagnosis and treatment considerations for women with COPD. Int J Clin Pract 2009; 63: 486-493.

9 Cote CG, Chapman KR. Diagnosis and treatment considerations for women with COPD. Int J Clin Pract 2009; 63: 486-493.

10 Mannino DM, Homa DM, Akinbami LJ, et al. Chronic obstructive pulmonary disease surveillance - United States, 1971-2000. MMWR Surveill Summ 2002; 51: 1-16.

11 Sanchez-Munoz G, Lopez-de-Andres A, Hernandez-Barrera V, et al. Bronchiectasis in patients hospitalized with acute exacerbation of COPD in Spain: influence on mortality, hospital stay, and hospital costs (2006-2014) according to gender. PLoS One 2019; 14: e0211222.

12 Sorheim IC, Johannessen A, Gulsvik A, et al. Gender differences in COPD: are women more susceptible to smoking effects than men? Thorax 2010; 65: 480-485.

13 Raghavan D, Varkey A, Bartter T. Chronic obstructive pulmonary disease: the impact of gender. Curr Opin Pulm Med 2017; 23: 117-123.

14 Belo LF, Rodrigues A, Paes T, et al. Functional status of patients with COPD assessed by London Chest Activity of Daily Living Scale: gender association and validity of a cutoff point. Lung 2019; 197: 509-516.

15 Miravitlles M, de la Roza C, Naberan K, et al. Attitudes toward the diagnosis of chronic obstructive pulmonary disease in primary care. Arch Bronconeumolol 2006; 42: 3-8. 
Delgado A, Saletti-Cuesta L, Lopez-Fernandez LA, et al. Gender inequalities in COPD decision-making in primary care. Respir Med 2016; 114: 91-96.

17 Ruiz MT, Verbrugge LM. A two way view of gender bias in medicine. J Epidemiol Community Health 1997; 51: $106-109$.

18 Delgado A, Saletti-Cuesta L, López-Fernández LA, et al. Gender inequalities in COPD decision-making in primary care. Respir Med 2016; 114: 91-96.

19 Schulz KF, Altman DG, Moher D, et al. CONSORT 2010 statement: updated guidelines for reporting parallel group randomised trials. BMJ; 2010: 340: c332.

20 Ahmadi Z, Sandberg J, Shannon-Honson A, et al. Is chronic breathlessness less recognised and treated compared with chronic pain? A case-based randomised controlled trial. Eur Respir J 2018; 52: 1800887.

21 Lisspers K, Larsson K, Janson C, et al. Gender differences among Swedish COPD patients: results from the ARCTIC, a real-world retrospective cohort study. NPJ Prim Care Respir Med 2019; 29: 45.

22 Arne M, Lisspers K, Ställberg B, et al. How often is diagnosis of COPD confirmed with spirometry? Respir Med 2010; 104: 550-556.

23 Nishi SPE, Wang Y, Kuo Y-F, et al. Spirometry use among older adults with chronic obstructive pulmonary disease: 1999-2008. Ann Am Thorac Soc 2013; 10: 565-573.

24 Watson L, Vestbo J, Postma DS, et al. Gender differences in the management and experience of chronic obstructive pulmonary disease. Respir Med 2004; 98: 1207-1213.

25 de Torres JP, Casanova C, de Garcini AM, et al. COPD heterogeneity: gender differences in the multidimensional BODE index. Int J Chron Obstruct Pulmon Dis 2007; 2: 151-155.

26 Fuller-Thomson E, Chisholm RS, Brennenstuhl S. COPD in a population-based sample of never-smokers: interactions among sex, gender, and race. Int J Chronic Dis 2016; 2016: 5862026.

27 Grabicki M, Kuznar-Kaminska B, Rubinsztajn R, et al. COPD course and comorbidities: are there gender differences? Adv Exp Med Biol 2019; 1113: 43-51.

28 Jia G, Lu M, Wu R, et al. Gender difference on the knowledge, attitude, and practice of COPD diagnosis and treatment: a national, multicenter, cross-sectional survey in China. Int J Chron Obstruct Pulmon Dis 2018; 13: 3269-3280.

29 Li X, Obeidat M, Zhou G, et al. Responsiveness to ipratropium bromide in male and female patients with mild to moderate chronic obstructive pulmonary disease. EBioMedicine 2017; 19: 139-145.

30 Henoch I, Strang S, Löfdahl C-G, et al. Management of COPD, equal treatment across age, gender, and social situation? A register study. Int J Chron Obstruct Pulmon Dis 2016; 11: 2681-2690.

31 Varkey AB. Chronic obstructive pulmonary disease in women: exploring gender differences. Curr Opin Pulm Med 2004; 10: 98-103.

32 Nguyen L-P, Beck E, Cayetano K, et al. Differential pulmonary rehabilitation outcomes in patients with and without COPD: ROLE OF GENDER. J Cardiopulm Rehabil Prev 2017; 37: 350-355.

33 Fernandez-Garcia S, Represas-Represas C, Ruano-Ravina A, et al. Social profile of patients admitted for COPD exacerbations. A gender analysis. Arch Bronconeumol 2019; 56: 84-89.

34 Global Initiative for Chronic Obstructive Lung Disease. Global Strategy for the Diagnosis, Management, and Prevention of Chronic Obstructive Pulmonary Disease: 2019 Report. www.goldcopd.org/wp-content/uploads/2018/ 11/GOLD-2019-v1.7-FINAL-14Nov2018-WMS.pdf.

35 Veloski J, Tai S, Evans AS, et al. Clinical vignette-based surveys: a tool for assessing physician practice variation. Am J Med Qual 2005; 20: 151-157. 